\title{
À propos d'un cas du syndrome de Lewis-Sumner observé au Niger
}

\author{
About a Case of Lewis-Sumner Syndrome Observed in Niger
}

\author{
H. Assadeck - M. Toudou Daouda - F. Hassane Djibo - D. Douma Maiga · É. Adehossi Omar \\ Reçu le 15 juillet 2018; accepté le 24 janvier 2019 \\ (C) Société de pathologie exotique et Lavoisier SAS 2019
}

Résumé Nous rapportons le premier cas documenté du syndrome de Lewis-Sumner (SLS) observé dans le service de neurologie de l'hôpital national de Niamey, Niger. Il s'agissait d'un homme de 53 ans, sans antécédents pathologiques connus, qui a présenté environ deux semaines après un syndrome pseudogrippal, des paresthésies dans la main droite dans le territoire du nerf médian. L'évolution s'est faite de façon rapidement progressive en deux semaines avec une atteinte sensitivomotrice asymétrique des quatre membres avec une prédominance aux membres supérieurs. L'étude électrophysiologique révélait la présence des blocs de conduction moteurs persistants, avec une diminution des amplitudes sensitives et des vitesses de conduction sensitive. L'étude du liquide céphalorachidien révélait une protéinorachie à $0,4 \mathrm{~g} / \mathrm{l}$, une glycorachie normale sans méningite. Après avoir éliminé certaines maladies systémiques et infectieuses pouvant expliquer le tableau clinique, le diagnostic du SLS a été établi chez notre patient sur la base des arguments cliniques et électrophysiologiques. Le patient fut traité par des immunoglobulines intraveineuses à la dose de $0,4 \mathrm{~g} / \mathrm{kg}$ par jour pendant cinq jours avec une bonne réponse clinique.

Mots clés Neuropathie sensitivomotrice inflammatoire démyélinisante multifocale Blocs de conduction nerveuse . Syndrome de Lewis-Sumner · Polyradiculonévrite inflammatoire démyélinisante chronique $\cdot$ Hôpital $\cdot$ Niamey Niger · Afrique intertropicale

H. Assadeck · M. Toudou Daouda $(\bowtie) \cdot$ F. Hassane Djibo Service de neurologie,

Hôpital national de Niamey, Niger

e-mail : moussatoudou@gmail.com

D. Douma Maiga · É. Adehossi Omar

Service de médecine interne,

Hôpital national de Niamey, Niger

H. Assadeck · D. Douma Maiga · É. Adehossi Omar

Faculté des sciences de la santé,

université Abdou-Moumouni de Niamey, Niger

\begin{abstract}
We report the first documented case of LewisSumner syndrome observed in the Department of Neurology of the National Hospital of Niamey, Niger. It was a 53-year-old man with no known past medical history, who presented paresthesia on the right hand in the median nerve territory about 2 weeks after a flu-like syndrome. The symptoms progressed rapidly in two weeks with asymmetric sensitivomotor involvement of the 4 limbs with a predominance to the upper limbs. The electrophysiological study revealed persistent motor conduction blocks with a decrease in sensory amplitudes and sensory conduction velocities. Cerebrospinal fluid study revealed a proteinorachia at $0.4 \mathrm{~g} / \mathrm{l}$ with normal glycorachia without meningitis. After ruling out other systemic and infectious diseases that explains the clinical picture, the diagnosis of Lewis-Sumner syndrome has been established in the patient based on clinical and electrophysiological arguments. The patient was treated with intravenous immunoglobulins $0.4 \mathrm{~g} / \mathrm{kg}$ per day during 5 days with a good clinical response.
\end{abstract}

Keywords Inflammatory demyelinating sensorimotor neuropathy $\cdot$ Nerve conduction blocks $\cdot$ Lewis-Sumner syndrome $\cdot$ Polyradiculonevritis · Hospital $\cdot$ Niamey Niger $\cdot$ Sub-Saharan Africa

\section{Introduction}

Décrit pour la première fois en 1982 par Lewis et al. [7], le syndrome de Lewis-Sumner (SLS) est une neuropathie sensitivomotrice inflammatoire démyélinisante multifocale à blocs de conduction persistants. Il se caractérise par une symptomatologie sensitivomotrice asymétrique, de systématisation tronculaire et d'évolution progressive ou par poussée-rémission, intéressant essentiellement les membres supérieurs [18]. C'est une variante rare de la polyradiculonévrite inflammatoire démyélinisante chronique (PIDC) [15,18]. 
Nous rapportons l'observation du premier cas documenté de SLS observé dans le service de neurologie de l'Hôpital national de Niamey, Niger.

\section{Présentation du cas}

Un homme de 53 ans, sans antécédents pathologiques connus, a présenté le 23 novembre 2017 des paresthésies dans la main droite dans le territoire du nerf médian, précédées environ deux semaines auparavant par un syndrome pseudogrippal. L'évolution s'est faite de façon rapidement progressive avec une atteinte sensitivomotrice des deux membres supérieurs en trois jours avec une prédominance à droite, puis des quatre membres en deux semaines avec une prédominance aux membres supérieurs. L'examen neurologique retrouvait un déficit moteur à quatre cinquième sur les fléchisseurs du poignet droit, les interosseux de la main gauche et le tibial antérieur droit, à trois cinquième sur les interosseux de la main droite et une abolition des réflexes ostéotendineux aux membres supérieurs. Le reste de l'examen neurologique était normal.

L'étude électrophysiologique du 12 décembre 2017 (soit 19 jours après le début des symptômes) révélait un allongement des latences distales motrices, une diminution des vitesses de conduction motrice et sensitive, une diminution des amplitudes motrices et sensitives, un allongement des ondes $F$ et la présence des blocs de conduction moteurs sur les nerfs médian, cubital et fibulaire droits (Tableau 1, Fig. 1).

Le bilan biologique, comportant une numération formule sanguine, une vitesse de sédimentation, un bilan rénal et hépatique, était sans anomalie particulière, ainsi que le dosage de la vitamine B12 et des folates sériques. L'étude $\mathrm{du}$ liquide céphalorachidien (LCR) révélait une

\begin{tabular}{|c|c|c|c|c|c|}
\hline Nerf stimulé & Site de stimulation & Latences (ms) & Amplitudes (mV) & $\begin{array}{l}\text { Vitesse de conduction } \\
(\mathrm{m} / \mathrm{s})\end{array}$ & Onde F (ms) \\
\hline \multicolumn{6}{|l|}{ Réponses motrices } \\
\hline \multirow[t]{3}{*}{ Médian droit } & Poignet & 5,68 & 6,87 & & 34,6 \\
\hline & Sous-coude & 12,08 & 7,35 & 45,27 & \\
\hline & Sus-coude & 15,14 & 0,68 & 24,58 & \\
\hline \multirow[t]{4}{*}{ Ulnaire droit } & Poignet & 2,85 & 6,6 & & 32,5 \\
\hline & Sous-coude & 8,88 & 6,4 & 48,1 & \\
\hline & Sus-coude & 12,2 & 6,4 & 51,2 & \\
\hline & Point d'Erb & 10,3 & 0,10 & 33,7 & \\
\hline \multirow[t]{3}{*}{ Médian gauche } & Poignet & 4,09 & 13,59 & & 34,9 \\
\hline & Sous-coude & 10,68 & 11,86 & 41,72 & \\
\hline & Sus-coude & 11,67 & 12,55 & 30,27 & \\
\hline \multirow[t]{3}{*}{ Ulnaire gauche } & Poignet & 2,79 & 6,5 & & 34,5 \\
\hline & Sous-coude & 8,18 & 6,3 & 53,8 & \\
\hline & Sus-coude & 11,5 & 5,8 & 54,2 & \\
\hline \multirow[t]{3}{*}{ Fibulaire droit } & Cheville & 4,53 & 6,4 & & 62,6 \\
\hline & Tête du péroné & 15,5 & 5,4 & 35,4 & \\
\hline & Sus-péroné & 16,3 & 3,1 & 29,7 & \\
\hline Tibial droit & Cheville & 5,83 & 5,3 & & 64,1 \\
\hline \multirow[t]{3}{*}{ Fibulaire gauche } & Cheville & 1,71 & 4,8 & & 62,6 \\
\hline & Tête du péroné & 15,3 & 4,2 & 32,4 & \\
\hline & Sus-péroné & 15,0 & 4,1 & 32,1 & \\
\hline Tibial gauche & Cheville & 7,79 & 5,7 & & 42,2 \\
\hline \multicolumn{6}{|l|}{ Réponses sensitives } \\
\hline Médian droit & Index & 2,25 & 13,6 & 34,3 & \\
\hline Ulnaire droit & Auriculaire & 2,46 & 10,3 & 42 & \\
\hline Médian gauche & Index & 3,5 & 17,9 & 40 & \\
\hline Ulnaire gauche & Auriculaire & 2,48 & 14 & 52 & \\
\hline Sural gauche & Jambe & 2,63 & 8,9 & & \\
\hline Musculocutané droit & Jambe & 2,27 & 10,4 & & \\
\hline
\end{tabular}


A

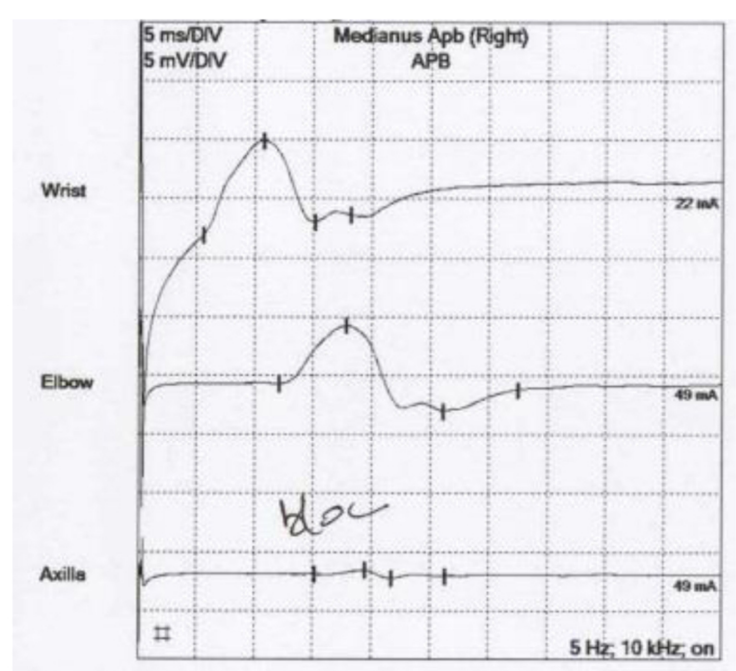

B

Fig. 1 A. Bloc de conduction moteur sur le nerf médian droit. B. Bloc de conduction moteur sur le nerf cubital droit au point d'Erb / $A$. Nerve conduction block on the right median nerve. B. Nerve conduction block on the right cubital nerve at the Erb's point

protéinorachie à $0,4 \mathrm{~g} / \mathrm{l}$, une glycorachie normale sans méningite. L'électrophorèse des protéines sériques, l'hémoglobine glyquée, la TSH et la T4 étaient normales. Les sérologies VIH, syphilis, Lyme, hépatites $\mathrm{B}$ et $\mathrm{C}$ étaient négatives. Les anticorps antinucléaires, anti-ADN natifs, les anticorps antiantigènes nucléaires solubles et le facteur rhumatoïde étaient normaux. La radiographie thoracique ne révélait aucune anomalie particulière. L'imagerie par résonance magnétique (IRM) médullaire ne montrait pas d'anomalie de signal sur la moelle.

Un traitement par immunoglobulines en intraveineux (IgIV) à la dose de $0,4 \mathrm{~g} / \mathrm{kg}$ par jour pendant cinq jours, associé à une kinésithérapie motrice, a permis une bonne amélioration clinique.

L'étude électrophysiologique du 12 mars 2018 (soit 16 semaines après le début des symptômes) révélait la persistance des blocs de conduction moteurs (Tableau 2).

Ainsi, le diagnostic du SLS fut retenu sur la base des arguments cliniques et électrophysiologiques.

\section{Discussion}

Nous rapportons le premier cas documenté du SLS observé dans le service de neurologie de l'Hôpital national de Niamey. L'installation des symptômes avait été rapidement progressive chez notre patient avec une atteinte sensitivomotrice asymétrique des quatre membres en deux semaines, avec une prédominance aux membres supérieurs. Cette installation aiguë des symptômes nous a fait discuter le diagnostic du syndrome de Guillain-Barré atypique. Le diagnostic du SLS a été établi sur la base des arguments cliniques et électrophysiologiques, et après avoir éliminé certaines maladies systémiques et infectieuses pouvant expliquer le tableau clinique. L'électroneuromyogramme et le bilan immunologique systémique non disponibles au Niger ont été réalisés hors du pays.

Le SLS est considéré comme une variante focale et asymétrique de la PIDC, affectant préférentiellement les hommes avec un pic de fréquence après l'âge de 40 ans $[15,17,18]$. La présentation clinique se caractérise par des troubles sensitifs et/ou moteurs à début unilatéral, préférentiellement aux membres supérieurs le plus souvent dans le territoire du nerf cubital ou médian [15]. Chez notre patient, le début des symptômes avait été marqué par des paresthésies de la main droite dans le territoire du nerf médian. Bien qu'elle soit peu fréquente, l'atteinte des nerfs crâniens a également été rapportée dans le SLS, notamment le nerf optique, les oculomoteurs, le trijumeau, le nerf facial $[7,15,17,18]$. Dans les formes évoluées du SLS, les troubles sensitifs ou moteurs peuvent devenir plus ou moins symétriques, et il devient difficile de déterminer si le patient est atteint du SLS ou de la PIDC $[6,9,15]$.

Les mécanismes physiopathologiques du SLS et de la PIDC restent encore méconnus. Cependant, la mise en évidence des cellules inflammatoires dans les biopsies nerveuses et l'élévation des taux sériques des cytokines et du TNF-alpha (tumor necrosis factor alpha) dans le SLS et la PIDC suggèrent une origine dysimmunitaire. En outre, la bonne amélioration clinique de ces pathologies sous IgIV, 


\begin{tabular}{|c|c|c|c|c|c|}
\hline Nerf stimulé & $\begin{array}{l}\text { Site } \\
\text { de stimulation }\end{array}$ & Latences (ms) & Amplitudes (mV) & $\begin{array}{l}\text { Vitesse } \\
\text { de conduction }(\mathrm{m} / \mathrm{s})\end{array}$ & Onde F (ms) \\
\hline \multicolumn{6}{|l|}{ Réponses motrices } \\
\hline \multirow[t]{3}{*}{ Médian droit } & Poignet & 4,16 & 1,97 & & 36,5 \\
\hline & Sous-coude & 11,0 & 1,44 & 46,8 & \\
\hline & Sus-coude & 12,6 & 0,90 & 34,58 & \\
\hline \multirow[t]{4}{*}{ Ulnaire droit } & Poignet & 3,38 & 3,9 & & 34,7 \\
\hline & Sous-coude & 9,08 & 3,9 & 56,1 & \\
\hline & Sus-coude & 12,2 & 3,5 & 41,7 & \\
\hline & Point d'Erb & 10,3 & 1,10 & 25,3 & \\
\hline \multirow[t]{3}{*}{ Médian gauche } & Poignet & 4,04 & 2,7 & & 34,5 \\
\hline & Sous-coude & 10,5 & 2,3 & 48,0 & \\
\hline & Sus-coude & 12,3 & 2,4 & 72,2 & \\
\hline \multirow[t]{4}{*}{ Ulnaire gauche } & Poignet & 2,65 & 5,0 & & 40,3 \\
\hline & Sous-coude & 9,23 & 4,2 & 47,1 & \\
\hline & Sus-coude & 13,1 & 3,1 & 31,0 & \\
\hline & Point d'Erb & 12,6 & 3,2 & 32,1 & \\
\hline \multirow[t]{3}{*}{ Fibulaire droit } & Cheville & 5,19 & 3,7 & & 63,9 \\
\hline & Tête du péroné & 16,2 & 2,6 & 36,3 & \\
\hline & Sus-péroné & 15,6 & 1,6 & 29,3 & \\
\hline Tibial droit & Cheville & 9,77 & 5,3 & & 73,5 \\
\hline \multirow[t]{3}{*}{ Fibulaire gauche } & Cheville & 5,1 & 4,0 & & 67,8 \\
\hline & Tête du péroné & 16,9 & 3,7 & 33,9 & \\
\hline & Sus-péroné & 15,8 & 3,2 & 33,4 & \\
\hline Tibial gauche & Cheville & 6,9 & 10,7 & & 68,1 \\
\hline \multicolumn{6}{|l|}{ Réponses sensitives } \\
\hline Médian droit & Index & 2,25 & 26,4 & 35,6 & \\
\hline Ulnaire droit & Auriculaire & 2,79 & 9,8 & 42 & \\
\hline Médian gauche & Index & 2,19 & 53,4 & 36,5 & \\
\hline Ulnaire gauche & Auriculaire & 2,71 & 14,0 & 52 & \\
\hline Musculocutané gauche & Jambe & 2,33 & 15,5 & & \\
\hline Musculocutané droit & Jambe & 3,06 & 10,8 & & \\
\hline
\end{tabular}

corticothérapie ou échanges plasmatiques constitue un argument de plus en faveur du mécanisme dysimmunitaire [14]. Kedra et al. [5] ont rapporté un cas de SLS survenu concomitamment avec une polyarthrite rhumatoïde, renforçant l'hypothèse de la théorie dysimmunitaire. Par ailleurs, des cas de SLS secondaires à un traitement anti-TNF-alpha (infliximab) ont été rapportés $[1,3,8,12]$. Le TNF-alpha est une cytokine pro-inflammatoire et immunorégulatrice, impliquée dans l'activation des macrophages, des lymphocytes $\mathrm{B}$ et $\mathrm{T}$, et la sécrétion d'autres cytokines proinflammatoires [1]. Considérant ces effets du TNF-alpha, on s'attendait aux effets anti-inflammatoires et immunomodulateurs des anti-TNF-alpha. Dans certaines situations, les anti-TNF-alpha sont susceptibles d'induire la synthèse des auto-anticorps dirigés contre les gangliosides et d'autres antigènes de surface des nerfs, entrainant par conséquent des neuropathies aiguës ou chroniques [3]. Le syndrome pseudogrippal précédant le début des symptômes, chez notre patient, suggère l'hypothèse d'une réaction immunologique croisée des auto-anticorps dirigés, l'agent infectieux interagissant avec les antigènes de surface des nerfs. Cette hypothèse reste à démontrer.

Sur le plan électrophysiologique, le SLS se caractérise par la présence des blocs de conduction persistants sur les nerfs moteurs et sensitifs atteints $[6,7,15,17]$. Il se distingue de la PIDC par une atteinte asymétrique segmentaire multitronculaire $[6,7,18]$. Dans certaines situations, seuls les potentiels évoqués sensitifs permettent de mettre en évidence l'atteinte sensitive en raison de l'accessibilité difficile au segment démyélinisé à l'électroneuromyographie [18].

Il est important également de distinguer le SLS de la neuropathie motrice multifocale à blocs de conduction 
(NMMBC). La NMMBC se différencie du SLS par l'absence de l'atteinte sensitive clinique, électrophysiologique et histologique, et par la forte positivité des anticorps IgM anti-GM1 [6,17,18].

La quasi-totalité des cas rapportés du SLS ont des anticorps IgM anti-GM1 négatifs [6]. Yang et al. [19] ont rapporté en 2004 un cas de SLS avec des anticorps IgM antiGM1 à un taux sérique très faible.

L'étude du LCR dans le SLS révèle une protéinorachie normale ou une élévation légère à modérée dépassant rarement $1 \mathrm{~g} / \mathrm{l}$, ce qui suggère que la démyélinisation est très focale, touchant plus fréquemment le segment nerveux moyen, et que les racines sont peu affectées $[6,7,18]$.

La biopsie nerveuse, non faite chez notre patient, montre des fibres démyélinisées ou faiblement myélinisées sans ou avec des infiltrats endoneuraux et périneuraux de macrophages et certains lymphocytes $B$ et $T$, sans signes histologiques de vascularite au niveau des vasa nervorum $[10,15,17]$. L'absence des cellules inflammatoires à l'étude histologique dans certains cas pourrait s'expliquer par le fait que le processus inflammatoire est supprimé par l'immunothérapie [10].

La biopsie nerveuse n'est pas un examen de routine dans le diagnostic des neuropathies inflammatoires démyélinisantes, mais elle peut aider lorsqu'elle est réalisée à éliminer certaines maladies telles que l'amylose, la sarcoïdose, les vascularites, etc. [3].

L'IRM, de préférence 3 Tesla, joue un rôle important dans l'étude anatomique du plexus brachial $[2,4,13]$. Elle peut être utile dans le diagnostic des neuropathies inflammatoires démyélinisantes telles que la PIDC, la NMMBC et le SLS, en complément à une étude électrophysiologique non concluante, par la mise en évidence des anomalies en faveur de démyélinisation au niveau des plexus brachial et/ou lombosacré $[2,4,13,16,17]$.

L'hypersignal et l'épaississement des racines ou troncs nerveux sur les séquences pondérées $\mathrm{T} 2$ sont les anomalies remnographiques rapportées, sans ou avec prise de gadolinium $[4,13,16,17]$. Dans le SLS et la NMMBC, les anomalies remnographiques sont asymétriques et corrélées aux signes cliniques et électrophysiologiques $[4,13]$.

Le traitement de première ligne du SLS repose sur les IgIV et la corticothérapie orale $[15,18]$. Les IgIV sont le plus souvent prescrites en première intention $[13,15,17,18]$. Le taux de réponse aux IgIV dans le SLS varie de 56 à $73 \%$ $[15,18]$. Chez les patients qui ne répondent pas aux IgIV après deux ou trois cures ou encore ceux qui sont dépendants aux IgIV, un traitement par prednisone ( $1 \mathrm{mg} / \mathrm{kg}$ par jour) peut être instauré pendant quatre à six semaines, suivi d'une dégression progressive [18]. Le taux de réponse à la corticothérapie est d'environ $50 \%[6,15]$. D'autres traitements peuvent être prescrits dans le SLS, notamment l'azathioprine, le cyclophosphamide, les échanges plasmatiques $[11,18]$.

\section{Conclusion}

Le diagnostic des affections du système nerveux périphérique reste encore un défi de diagnostique au Niger en raison de la non-disponibilité de l'électroneuromyogramme. L'absence de cas rapportés du SLS dans les années antérieures est probablement due au manque de moyens diagnostiques au Niger.

Liens d'intérêts : Les auteurs déclarent ne pas avoir de liens d'intérêts.

\section{Références}

1. Cirillo G, Todisco V, Tedeschi G (2016) Lewis-Sumner syndrome associated with infliximab therapy in ulcerative colitis. Neurol Sci 37:1005-8. doi: 10.1007/s10072-016-2490-4

2. Haakma W, Jongbloed BA, Froeling M, et al (2017) MRI shows thickening and altered diffusion in the median and ulnar nerves in multifocal motor neuropathy. Eur Radiol 27:2216-24. doi: 10.1007/s00330-016-4575-0

3. Hooper DR, Tarnopolsky MA, Baker SK (2008) Lewis-Sumner syndrome associated with infliximab therapy in rheumatoid arthritis. Muscle Nerve 38:1318-25. doi: 10.1002/mus.21051

4. Jongbloed BA, Bos JW, Rutgers D, et al (2017) Brachial plexus magnetic resonance imaging differentiates between inflammatory neuropathies and does not predict disease course. Brain Behav 7: e00632. doi: $10.1002 / \mathrm{brb3} .632$

5. Kedra J, Foltz V, Viala K, et al (2017) Lewis-Sumner syndrome in a patient with rheumatoid arthritis: link between rheumatoid arthritis and demyelinating polyradiculoneuropathies. Joint Bone Spine 84:485-7. doi: 10.1016/j.jbspin.2017.02.013

6. Lewis RA (2007) Neuropathies associated with conduction block. Curr Opin Neurol 20:525-30. doi: 10.1097/WCO.0b013e3282efa143

7. Lewis RA, Sumner AJ, Brown MJ, Asbury AK (1982) Multifocal demyelinating neuropathy with persistent conduction block. Neurology 32:958-64

8. Nancey S, Bouhour F, Boschetti G, et al (2010) Lewis-Sumner syndrome following infliximab treatment in Crohn's disease: a report of 2 cases. Inflamm Bowel Dis 16:1450-3. doi: $10.1002 / \mathrm{ibd} .21202$

9. Oh SJ, Claussen GC, Kim DS (1997) Motor and sensory demyelinating mononeuropathy multiplex (multifocal motor and sensory demyelinating neuropathy): a separate entity or a variant of chronic inflammatory demyelinating polyneuropathy? J Peripher Nerv Syst 2:362-9

10. Oh SJ, LaGanke C, Powers R, et al (2005) Multifocal motor sensory demyelinating neuropathy: inflammatory demyelinating polyradiculoneuropathy. Neurology 65:1639-42. doi: 10.1212/01.wnl. $0000184592.54972 .5 \mathrm{e}$

11. Park YE, Yook JW, Kim DS (2010) A case of Lewis-Sumner syndrome showing dramatic improvement after plasma exchange. J Korean Med Sci 25:1101-4. doi: 10.3346/jkms.2010.25.7.1101

12. Poisson A, Bouhour F, Nancey S, Gervais H (2007) Le syndrome de Lewis-Sumner : un effet indésirable des anti-TNF-alpha ? Rev Neurol 163:104. https://doi.org/10.1016/S0035-3787(07)90685-2

13. Rajabally YA, Knopp MJ, Martin-Lamb D, Morlese J (2014) Diagnostic value of MR imaging in the Lewis-Sumner syndrome: a case series. J Neurol Sci 342:182-5. doi: 10.1016/ j.jns.2014.04.033 
14. Ramchandren S, Lewis RA (2009) Chronic neuropathies - chronic inflammatory demyelinating neuropathy and its variants. Front Neurol Neurosci 26:12-25. doi: 10.1159/000212313

15. Saperstein DS, Amato AA, Wolfe GI, et al (1999) Multifocal acquired demyelinating sensory and motor neuropathy: the Lewis-Sumner syndrome. Muscle Nerve 22:560-6

16. Van Es HW, Van den Berg LH, Franssen H, et al (1997) Magnetic resonance imaging of the brachial plexus in patients with multifocal motor neuropathy. Neurology 48:1218-24
17. Van den Berg-Vos RM, Van den Berg LH, Franssen H, et al (2000) Multifocal inflammatory demyelinating neuropathy: a distinct clinical entity? Neurology 54:26-32

18. Viala K, Renié L, Maisonobe T, et al (2004) Follow-up study and response to treatment in 23 patients with Lewis-Sumner syndrome. Brain 127:2010-7

19. Yang YW, Liu CH, Tsai $\mathrm{CH}$, et al (2004) Multifocal acquired demyelinating sensory and motor neuropathy: report of a case and review of the literature. Acta Neurol Taiwan 13:24-8 